describes how the properties of quantum systems can be tied together. If these behaviours can be more precisely controlled, they would generate exponential gains in processing power for certain tasks compared with today's supercomputers. And that is what the team at Google has achieved.

Its chip, known as Sycamore, comprises just 53 individually controllable superconducting quantum bits (qubits), the basic building blocks of quantum computers. The team chose to calculate the outputs of a random quantum circuit - rather like a quantum random number generator. This is not an easy problem, and the Summit supercomputer at Oak Ridge National Laboratory in Tennessee, the world's most powerful machine in its class, would have taken 10 millennia to complete it, the researchers say. Sycamore needed only 200 seconds.

Summit can call on more than 9,000 of the most powerful central processing units ( 8 billion transistors in each) and nearly 28,000 graphics processors ( 21 billion transistors each). With such raw computing power outgunned by just 53 qubits, it's understandable that quantum computers are generating such excitement and optimism.

But this demonstration of quantum supremacy is extremely limited. There's a vast gap to be bridged before quantum computers can do more meaningful things - such as simulating the properties of materials or chemical reactions, or accelerating drug discovery.

For one thing, quantum computers are highly sensitive to environmental noise - including everyday phenomena such as temperature changes and electromagnetic fields. And researchers are a long way from being able to design out these and other obstacles.

Instead of proceeding with caution, a quantum gold rush is under way, with investors joining governments and companies to pour large sums of money into developing quantum technologies. Unrealistic expectations are being fuelled that powerful general-purpose quantum computers could soon be on the horizon. Such misguided optimism could be dangerous for the future of this still-fledgling field.

Such a landscape has created a flourishing network of quantum technologists, but those providing the funding will eventually seek a return on investment. There are already concerns that some firms are over-promising, which is why over-hyping this landmark demonstration could raise expectations further. Researchers fear that, if quantum computers fail to deliver anything useful soon, a 'quantum winter' could descend in which research progress slows, investment stalls and disillusion sets in.

The powerful processors that underpin today's devices such as smartphones were developed from decades of sustained investment - often public investment - in research. Quantum processors will similarly require what innovation economists call 'patient capital'.

Too often in the history of science and technology, expectations are raised, only for reality to get in the way. Quantum computers are still near the start of a long and unpredictable journey. As they encounter challenges and costs start to mount, researchers must know that they can reach their destination.

\title{
Young universities show leadership
}

\section{Thriving new institutions can share lessons in building research and publishing capacity.}

$\mathrm{T}$ his week, an analysis from Nature Research's Nature Index team (see supplement, page S53) looks at the contribution of 'young' universities to research publishing in the natural sciences. Young universities - those aged 50 years or less - are quickly establishing a reputation in teaching and research. However, in Africa, more needs to be done to build their capacity.

The analysis looked at the contributions of authors from 100 young universities in 2018 to 82 journals in the natural sciences. The journals were chosen by an independent panel of researchers, and span the life sciences, physical sciences, chemistry, and Earth and environmental sciences. Author contribution was recorded in several ways, including the total number of articles published by an institution's affiliated researchers, as well as the share of each institution's contribution to those articles.

In most assessments of research-intensive universities, those in the United States and Europe tend to dominate. But among the leading 100 younger universities, there is much more of an east-west mix, spread across China (11 universities), Germany (11), India (10), Australia (9), South Korea (8) and the United States (8).

Authors from the University of the Chinese Academy of Sciences in Beijing are by far the most prolific, contributing 1,816 articles to the listed journals. That is on a par with the number of articles from older institutions in the United States, Europe and Japan, and substantially ahead of second-placed Nanyang Technological University in Singapore (569 articles).

The absence of institutions from Africa in the analysis is partly because many authors there publish in journals that the index does not capture, including in fields such as agriculture, water resources, primary health care and education. But a comparative lack of financial resources for researchers in the natural sciences is also a factor.

In the spirit of south-south collaboration, universities recognized for their publishing in the natural sciences have an opportunity to support those in need of a boost. Many of the young universities assessed in the index are in countries that, even one generation ago, were at an earlier stage of development. They will have valuable lessons to pass on in building research and publishing capacity.

China's fast-expanding universities are already doing this through the Belt and Road Initiative. Rising institutions in other countries, too, will find mutual benefits by sharing experiences and working with research partners in Africa and elsewhere in the global south. 